\title{
Astaxanthin prevents and reverses the activation of mouse primary hepatic stellate cells
}

Yue Yang ${ }^{1}$, Minkyung Bae ${ }^{1}$, Bohkyung Kim ${ }^{1}$, Young-Ki Park ${ }^{1}$, Sung I. Koo ${ }^{1}$, Ji-Young Lee ${ }^{1,2,3}$

${ }^{1}$ Department of Nutritional Sciences, ${ }^{2}$ Institute of Systems Genomics, University of Connecticut, Storrs, Connecticut 06269, USA

${ }^{3}$ Corresponding author: Ji-Young Lee, Ph.D.

Phone: 860-486-1827Ｆax: 860-486-3674Ｅmail: ji-young.lee@uconn.edu

\begin{abstract}
Abbreviations: ADRP, adipocyte differentiation-related protein; $\alpha$-SMA, $\alpha$-smooth muscle actin; ASTX, astaxanthin; Col1A1, procollagen type I, alpha 1; ECM, extracellular matrix; GCL, glutamate-cysteine ligase catalytic subunit; HSC, hepatic stellate cell; aHSC, activated HSC; iHSC, inactivated HSC; qHSC, quiescent HSC; LRAT, lecithin retinol acyltransferase; NAFLD, non-alcoholic fatty liver disease; NASH, non-alcoholic steatohepatitis; NOX4, NADPH oxidase 4; Nrf2, Nuclear erythroid 2-related factor 2; qRT-PCR, quantitative realtime PCR; ROS, reactive oxygen species; SREBP1c, sterol regulatory element binding protein 1c; tBHP, tert-butyl hydrogen peroxide; TGF $\beta 1$, transforming growth factor $\beta 1$.
\end{abstract}




\begin{abstract}
Activation of hepatic stellate cells (HSCs) is a critical step that leads to the development of liver fibrosis. We showed that astaxanthin (ASTX), a xanthophyll carotenoid, displays anti-fibrogenic effects in LX-2 cells, a human HSC cell line. In this study, we further determined the effect of ASTX on HSC activation and inactivation using primary HSCs from C57BL/6J mice. Quiescent and activated HSCs were incubated with ASTX $(25 \mu \mathrm{M})$ at different stages of activation. ASTX prevented the activation of quiescent HSCs, as evidenced by the presence of intracellular lipid droplets and reduction of $\alpha$-smooth muscle actin ( $\alpha$-SMA), a HSC activation marker. Also, ASTX reverted activated HSCs to a quiescent phenotype with the reappearance of lipid droplets with a concomitant increase in lecithin retinol acyltransferase mRNA. Cellular accumulation of reactive oxygen species was significantly reduced by ASTX, which was attributable to a decrease in NADPH oxidase 2 expression. The anti-fibrogenic effect of ASTX was independent of nuclear erythroid 2-related factor 2 (NRF2) as it was observed in HSCs from wild-type and $\mathrm{Nrf}_{2}{ }^{-/}$mice. In conclusion, ASTX inhibits HSC activation and reverts activated HSCs to a quiescent state. Further investigation is warranted to determine if ASTX effectively prevent the development of liver fibrosis.
\end{abstract}

Keywords: Astaxanthin; Liver fibrosis; Hepatic Stellate Cells; Reversal of hepatic stellate cell activation 


\section{Introduction}

Non-alcoholic fatty liver disease (NAFLD) is the most common cause of chronic liver diseases in the developed countries [1]. Fatty liver is susceptible to oxidative stress and inflammation, resulting in the progression of simple steatosis to non-alcoholic steatohepatitis (NASH) [2]. In response to oxidative stress, hepatocyte death, and inflammation, the liver initiates a wound-healing response by producing extracellular matrix (ECM) [3]. Excessive deposition of ECM leads to liver fibrosis. Although fibrosis is a reversible process, it can slowly advance to cirrhosis, which is considered irreversible and disrupts normal liver functions [4].

Hepatic stellate cells (HSCs) are primarily responsible for ECM production and fibrosis development in the liver [5]. In the normal liver, quiescent HSCs (qHSCs) are present in the space of Disse and contain cytoplasmic lipid droplets mainly consisting of retinyl esters [6]. In response to injury, qHSCs transdifferentiate to myofibroblast-like cells, which highly express $\alpha$ smooth muscle actin ( $\alpha$-SMA), a myofibroblast marker, and ECM proteins, e.g., procollagen type I $\alpha 1$ (Col1A1). The activated HSCs (aHSCs) are highly proliferative and migrate to the sites of injury. Recent studies have suggested that HSC activation is a reversible process [7]. When stimulants, such as oxidants or fibrogenic cytokines, are removed, aHSCs are either eliminated through apoptosis or reverted to an inactive phenotype, i.e., inactivated HSCs (iHSCs), which share common features with qHSCs but differ in their sensitivity to fibrogenic insults [7].

Astaxanthin (ASTX) is a xanthophyll carotenoid that is abundant in red marine animals, such as salmon and shrimp [8]. It is known as a potent antioxidant [9]. Our previous study demonstrated that ASTX prevents fibrogenic responses and accumulation of reactive oxygen species (ROS) induced by transforming growth factor $\beta 1$ (TGF $\beta 1$ ), a fibrogenic cytokine, in LX2 cells, a human HSC cell line [10]. LX-2 cells are commonly used as a HSC cell model, but 
they exhibit a minimal characteristics of a quiescent phenotype [11]. Therefore, the present study used primary mouse HSCs, which are in a quiescent state and can be transdifferentiated to aHSCs in culture, to determine the anti-fibrogenic effect of ASTX.

\section{Materials and methods}

\subsection{Cell culture}

Mouse primary HSCs were isolated from C57BL/6J, nuclear erythroid 2-related factor 2 (NRF2) knockout $\left(\mathrm{Nrf}^{-/}\right)$, and wild-type mice using a pronase/collagenase digestion method as described [10]. The cells were then cultured on untreated petri dishes (BD Falcon, Franklin Lakes, NJ), which is a well-established condition for HSC activation in vitro [12], and maintained in a low-glucose DMEM medium supplemented with 10\% FBS, 4 mM L-glutamine, and $\mathrm{P} / \mathrm{S}(100 \mathrm{U} / 100 \mu \mathrm{g}$ per $\mathrm{mL})$. Cells were cultured in a $37^{\circ} \mathrm{C}$ humidified cell culture chamber with $5 \% \mathrm{CO}_{2}$. All cell culture supplies were purchased from HyClone unless stated otherwise (Thermo Scientific, Logan, UT).

\subsection{ASTX treatment}

ASTX, provided by Fuji Chemical Industry Co., Ltd. (Toyama, Japan), was solubilized in DMSO as described [10]. After plated on an uncoated dish, mouse qHSCs were incubated with $25 \mu \mathrm{M}$ ASTX during activation. aHSCs were prepared by culturing qHSCs on an uncoated dish for $6 \mathrm{~d}$, were incubated with $25 \mu \mathrm{M}$ ASTX for 2 or 4 more days. Also, cells were incubated with $3 \mu \mathrm{M}$ tert-butyl hydrogen peroxide (tBHP, Thermo Scientific) for $12 \mathrm{~h}$ with or without 25 $\mu \mathrm{M}$ ASTX. Medium containing ASTX was replenished daily and DMSO vehicle control was run 
in parallel. Hereafter, "Xd + Yd" indicates that HSCs were activated for X days followed by ASTX treatment for Y days. Experimental scheme is shown in Figure 1.

\subsection{Quantitative realtime $P C R(q R T-P C R)$}

Total RNA extraction, cDNA synthesis, and qRT-PCR were conducted using a Bio-Rad CFX96 Real-Time system (Bio-Rad, Hercules, CA) as previously described $[13,14]$. Primer sequences will be available upon request.

\subsection{Western blot analysis}

Whole cell lysates and human tissue protein extracts were prepared and Western blot analysis were performed as described [14] using antibodies against $\alpha-$ SMA (Sigma, St. Louis, MO) and $\beta$-tubulin (Santa Cruz) was used as a loading control.

\subsection{ROS measurement}

Mouse primary qHSCs were seeded on an uncoated black 24-well $\mu$-Plate (Ibidi, Verona, WI). At day 6 of activation, cellular ROS levels were measured using dichlorofluorescin (SigmaAldrich, St. Louis, MO) as described [15]. The cells were then fixed with $4 \%$ formaldehyde for 10 min, and incubated with $0.1 \%$ CellTag 700 stain (Li-COR, Lincoln, NE), a fluorescent stain,

for cell number normalization, for $1 \mathrm{~h}$. Fluorescent signals were obtained by an Odyssey ${ }^{\circledR}$ CLx Infrared Imaging System (Li-COR) and a Li-COR Image Studio software. Data were expressed as relative fluorescent intensity divided by total cell stain signal. 


\subsection{Statistical analysis}

One-way analysis of variance with Newman Keul's pairwise comparison, or unpaired ttest was used to determine significant differences between groups by GraphPad Prism6 (GraphPad Software, La Jolla, CA). P values less than 0.05 were considered significant and all values were presented as mean \pm SEM.

\section{Results}

\subsection{ASTX inhibited the activation of qHSCs}

To investigate the inhibitory effect of ASTX on the activation of qHSCs, mouse primary HSCs were cultured on plastic substratum for $6 \mathrm{~d}$ in the absence or presence of ASTX. The expression of $\alpha$-SMA was markedly induced during activation in controls, whereas ASTX significantly decreased their mRNA (Figure 2A). Protein levels of $\alpha$-SMA were also noticeably decreased when HSCs were treated with ASTX for 2 days during activation (Figure 2B). At days 2 and 4, no noticeable differences in cell morphology were observed between control and ASTXtreated cells. However, at day 6, control cells displayed stretched, myofibroblast-like appearance with few cytoplasmic lipid droplets whereas the cells treated with ASTX for 2-6 days displayed more lipid droplets than controls (Figure 3).

\subsection{ASTX reverted aHSCs to $i H S C s$}

To examine if ASTX can revert aHSCs to iHSCs, aHSCs were incubated with ASTX for 2 or $4 \mathrm{~d}$. While control aHSCs displayed a myofibroblast-like appearance, there was reappearance of cytoplasmic lipid droplets in the ASTX-treated HSCs (Figure 4A). Furthermore, 
ASTX significantly decreased $\alpha$-SMA and Col1A1 mRNA (Figure 4B) and $\alpha$-SMA protein (Figure 4C). As lipid droplets reappeared when aHSCs were incubated with ASTX, we examined the ability of ASTX to alter the expression of genes that facilitate lipid droplet formation. ASTX significantly decreased the mRNA levels of sterol regulatory element binding protein 1c (SREBP1c), a transcriptional factor critical for lipogenesis [16], but markedly increased lecithin retinol acyltransferase (LRAT) mRNA, an enzyme that esterifies retinol to retinyl esters (Figure 4D). There was no significant difference in mRNA levels of adipocyte differentiation-related protein (ADRP), a lipid droplet surface protein [17], between control and ASTX-treated cells.

\subsection{ASTX prevented cellular ROS accumulation independent of NRF2 in HSCs}

As ROS can activate HSCs [18, 19], we evaluated if ASTX can reduce cellular ROS levels. ASTX treatment for $6 \mathrm{~d}$ during the activation of mouse primary HSC significantly lowered cellular ROS levels (Figure 5A). NADPH oxidase 2 (NOX2) and NOX4 have been shown to produce ROS in HSCs, activating the cells $[20,21]$. When ASTX was added at day 4 for $2 \mathrm{~d}$ during HSC activation, ASTX significantly increased NOX4 mRNA but decreased NOX2 mRNA (Figure 5B).

The activation of NRF2, a transcription factor for endogenous antioxidant defense, has been suggested to ameliorate liver fibrosis [22]. Also, we previously observed that ASTX induced the hepatic expression of NRF2 and its target antioxidant genes in vivo [23]. Therefore, we determined if the inhibitory action of ASTX in HSC activation is mediated via NRF2. When HSCs isolated from wild-type and $\mathrm{Nrf}^{-/-}$mice were activated for $6 \mathrm{~d}, \alpha$-SMA mRNA was not significantly different between HSCs from wild-type and $\mathrm{Nrf}^{-/-}$mice, while there was a 
significantly lower Col1A 1 in $\mathrm{Nrf}^{-/}$HSCs than wild-type (Figure 6A). Both in wild-type and $\mathrm{Nrf2}^{-/}$HSCs, ASTX was able to decrease $\alpha-S M A$ and Col1A1 mRNA. As NRF2 may be required when HSCs are under oxidative stress, HSCs from wild-type and $\mathrm{Nrf}^{-/}$mice were treated with tBHP at day 6 for $12 \mathrm{~h}$ in the presence or absence of ASTX. In both wild-type and $\mathrm{Nrf}^{-/}$HSCs, tBHP increased $\alpha$-SMA expression, whereas ASTX markedly reduced its expression. NRF2 expression was significantly inhibited by ASTX in wild-type HSCs (Figure 6B).

\section{Discussion}

Fibrosis is a key feature of NASH [24]. Although fibrogenesis is a necessary process for wound-healing, dysregulation of fibrogenic activity of HSCs can lead to liver fibrosis [12, 25]. Studies have shown that during fibrogenesis resolution, HSCs are removed by apoptosis or inactivated to a nearly-quiescent phenotype [26]. We previously demonstrated that ASTX has anti-fibrogenic effects in LX-2 cells. In the present study, using mouse primary HSCs, we evaluated the role of ASTX in the regulation of activation and inactivation of HSCs. Our data here showed that ASTX not only prevented the activation of qHSC but also facilitated the reversion of aHSC to an inactivated state.

In a healthy liver, qHSCs store retinoids in the cytosol and maintain an equilibrium between ECM production and degradation [27]. Upon liver injury, HSCs transdifferentiate to myofibroblasts and express $\alpha$-SMA and ECM proteins at high abundance. When qHSCs are plated on an uncoated plastic surface, they undergo "spontaneous activation" and are fully activated within 7 days with similar characteristics of HSCs shown in vivo during liver injury [12]. We observed that ASTX inhibited this activation process, as demonstrated by the presence 
of lipid droplets and inhibition of the expression of fibrogenic markers. This effect of ASTX may be explained by its potent antioxidant property [28, 29]. ROS, produced by damaged hepatocytes, Kupffer cells and aHSCs, are known to activate HSCs and increase ECM production [18, 19]. We observed that ASTX decreased cellular ROS accumulation in HSCs. Studies have shown that NOX2 and NOX4 are major superoxide-producing enzymes in HSCs [20, 21] and their expression is induced during HSC activation in vitro [30, 31]. In the present study, ASTX increased NOX4 mRNA, while it repressed NOX2 mRNA, suggesting that the decrease in cellular ROS by ASTX may be mediated in part by diminished expression of NOX2, but not NOX4. NOX2 and NOX4 have been shown to be induced by distinct cytokines via different transcription factors [32]. Therefore, further work is needed to determine how ASTX differentially alters the expression of NOX2 and NOX4 in HSCs.

As a master transcriptional regulator of endogenous antioxidant system, NRF2 senses cellular oxidative stress and induces antioxidant enzymes and glutamate-cysteine ligase catalytic subunit (GCLc) [33]. It has been shown that GCLc expression decreases during the activation of rat primary HSCs and increases when HSCs are inactivated [34]. Therefore, we determined a potential role of NRF2 in HSC activation. In the present study, the expression of $\alpha$-SMA and Col1A1 was not affected in $\mathrm{Nrf}^{-/}$HSCs relative to wild-type HSCs regardless of the presence of tBHP. In contrast, ASTX inhibited $\alpha$-SMA and Col1 A1 expression similarly in both wild-type and $\mathrm{Nrf}^{-/} \mathrm{HSCs}$, suggesting that the repression of the fibrogenic genes by ASTX is likely independent of NRF2. We also observed that $\alpha$-SMA expression is reduced in $\mathrm{Nrf}^{-\digamma} \mathrm{HSCs}$ relative to wild-type counterparts regardless of the presence or absence of tBHP. A recent study showed that the activation of NRF2 attenuates Col1A1 expression by repressing SMAD3 pathway in TGF $\beta 1$-stimulated renal fibroblastic cells [35]. However, there are no published 
reports that address a direct effect of NRF2 on the fibrogenic gene expression in aHSCs. Our data suggest that, at least in mouse primary HSCs activated on plastic substratum, the deficiency of NRF2 does not impact HSC activation.

Recent evidence shows that liver fibrosis is reversible when fibrogenic stimulants are cleared [36]. The reversal of liver fibrosis is accompanied by a reduction in the number of aHSCs by apoptosis [37] or inactivation of aHSCs to a nearly quiescent phenotype, i.e., iHSCs. iHSCs are characterized by the presence of cytosolic lipid droplets and diminished expression of fibrogenic markers, but iHSCs differ from qHSCs because they are more sensitive to fibrogenic stimulus than qHSCs [36]. When aHSCs were incubated with ASTX, we observed that cytoplasmic lipid droplets reappeared with a concomitant decrease in $\alpha$-SMA and ColA1 mRNA, suggesting that aHSCs were reverted to iHSCs. Studies have suggested that lipogenic genes, such as SREBP-1c and peroxisome proliferator-activated receptor $\gamma$, contribute to the inactivation of aHSCs [26]. However, we observed a decrease in SREBP-1c mRNA when aHSCs were incubated with ASTX with no significant change in the expression of ADRP, a surface protein present in lipid droplets in HSCs [38]. Instead, ASTX significantly increased the expression of LRAT [38]. LRAT-deficient mice completely lack lipid droplets in HSCs, suggesting a critical role of LRAT in lipid droplet formation in HSCs, although the LRATdeficient mice did not have elevated fibrogenesis [39]. Moreover, increased LRAT expression in mice has been shown to increase hepatic retinoid storage and inhibit liver fibrosis [40]. Therefore, our finding here suggests that ASTX may facilitate the reappearance of lipid droplets via the induction of LRAT expression, independent of SREBP-1c pathways. The expression of LRAT has been suggested to be co-regulated by GATA transcription factors and retinoic acid receptors, 
which are activated by retinoic acid [41]. Whether ASTX activates this transcription factor to induce LRAT is currently unknown.

In conclusion, our findings indicate that ASTX inhibits the activation of qHSCs and also reverts aHSCs to an inactivate state in mouse primary cells. Although further work is needed to elucidate the underlying mechanism, the present study provides novel evidence that ASTX is anti-fibrogenic and inhibits HSC activation. This is the first study to our knowledge that shows ASTX, a dietary component, can not only prevent HSC activation, but also revert the activated HSCs to a quiescent state. Further studies are warranted to explore its potential use for the prevention and treatment of liver fibrosis and fatty liver disease.

\section{Author contributions}

Y. Yang, M. Bae, B. Kim, and Y. Park conducted experiments; Y. Yang contributed to manuscript preparation; S. I. Koo and J. Lee contributed to study design, data interpretation and manuscript preparation.

\section{Acknowledgements}

All authors read this manuscript and claim no conflicts of interest. This work was supported by USDA AFRI 2012-67018-19290 and Fuji Chemical Industry Co. to J. Lee, and USDA Multistate/Hatch CONS00916 to S. I. Koo and J. Lee. 


\section{References}

[1] Lim YS, Kim WR. The global impact of hepatic fibrosis and end-stage liver disease. Clin Liver Dis. 2008;12:733-46.

[2] Kim D, Kim WR, Kim HJ, Therneau TM. Association between noninvasive fibrosis markers and mortality among adults with nonalcoholic fatty liver disease in the United States. Hepatology. 2013;57:1357-65.

[3] Bataller R, Brenner DA. Liver fibrosis. J Clin Invest. 2005;115:209-18.

[4] Ekstedt M, Franzén LE, Mathiesen UL, Thorelius L, Holmqvist M, Bodemar G, et al. Longterm follow-up of patients with NAFLD and elevated liver enzymes. Hepatology. 2006;44:86573.

[5] Hernandez-Gea V, Friedman SL. Pathogenesis of liver fibrosis. Annu Rev Pathol. 2011;6:425-56.

[6] Senoo H, Yoshikawa K, Morii M, Miura M, Imai K, Mezaki Y. Hepatic stellate cell (vitamin A-storing cell) and its relative--past, present and future. Cell Biol Int. 2010;34:1247-72.

[7] Kisseleva T, Brenner DA. Inactivation of myofibroblasts during regression of liver fibrosis. Cell Cycle. 2013;12:381-2.

[8] Higuera-Ciapara I, Felix-Valenzuela L, Goycoolea FM. Astaxanthin: a review of its chemistry and applications. Crit Rev Food Sci Nutr. 2006;46:185-96.

[9] Yang Y, Kim B, Lee J-Y. Astaxanthin structure, metabolism, and health benefits. J Hum Nutr Food Sci. 2013;1:1-1003.

[10] Yang Y, Kim B, Park Y-K, Koo SI, Lee J-Y. Astaxanthin prevents TGFß1-induced profibrogenic gene expression by inhibiting Smad3 activation in hepatic stellate cells. Biochim Biophys Acta. 2015;1850:178-85.

[11] Xu L, Hui AY, Albanis E, Arthur MJ, O'Byrne SM, Blaner WS, et al. Human hepatic stellate cell lines, LX-1 and LX-2: new tools for analysis of hepatic fibrosis. Gut. 2005;54:142-51.

[12] Friedman SL. Hepatic stellate cells: protean, multifunctional, and enigmatic cells of the liver. Physiol Rev. 2008;88:125-72.

[13] Park YK, Rasmussen HE, Ehlers SJ, Blobaum KR, Lu F, Schlegal VL, et al. Repression of proinflammatory gene expression by lipid extract of Nostoc commune var sphaeroides Kutzing, a blue-green alga, via inhibition of nuclear factor-kappaB in RAW 264.7 macrophages. Nutr Res. 2008;28:83-91. 
[14] Rasmussen HE, Blobaum KR, Park YK, Ehlers SJ, Lu F, Lee JY. Lipid extract of Nostoc commune var. sphaeroides Kutzing, a blue-green alga, inhibits the activation of sterol regulatory element binding proteins in HepG2 cells. J Nutr. 2008;138:476-81.

[15] Lee SG, Kim B, Yang Y, Pham TX, Park YK, Manatou J, et al. Berry anthocyanins suppress the expression and secretion of proinflammatory mediators in macrophages by inhibiting nuclear translocation of NF-kappaB independent of NRF2-mediated mechanism. J Nutr Biochem. 2014;25:404-11.

[16] Ferré P, Foufelle F. Hepatic steatosis: a role for de novo lipogenesis and the transcription factor SREBP-1c. Diabetes, Obesity and Metabolism. 2010;12:83-92.

[17] Lee TF, Mak KM, Rackovsky O, Lin YL, Kwong AJ, Loke JC, et al. Downregulation of hepatic stellate cell activation by retinol and palmitate mediated by adipose differentiationrelated protein (ADRP). J Cell Physiol. 2010;223:648-57.

[18] Nieto N, Friedman SL, Greenwel P, Cederbaum AI. CYP2E1-mediated oxidative stress induces collagen type I expression in rat hepatic stellate cells. Hepatology. 1999;30:987-96.

[19] Svegliati-Baroni G, Saccomanno S, van Goor H, Jansen P, Benedetti A, Moshage H. Involvement of reactive oxygen species and nitric oxide radicals in activation and proliferation of rat hepatic stellate cells. Liver. 2001;21:1-12.

[20] Jiang JX, Venugopal S, Serizawa N, Chen X, Scott F, Li Y, et al. Reduced nicotinamide adenine dinucleotide phosphate oxidase 2 plays a key role in stellate cell activation and liver fibrogenesis in vivo. Gastroenterology. 2010;139:1375-84.

[21] Sancho P, Mainez J, Crosas-Molist E, Roncero C, Fernández-Rodriguez CM, Pinedo F, et al. NADPH Oxidase NOX4 Mediates Stellate Cell Activation and Hepatocyte Cell Death during Liver Fibrosis Development. PLoS ONE. 2012;7:e45285.

[22] Yang JJ, Tao H, Huang C, Li J. Nuclear erythroid 2-related factor 2: a novel potential therapeutic target for liver fibrosis. Food Chem Toxicol. 2013;59:421-7.

[23] Yang Y, Seo JM, Nguyen A, Pham TX, Park HJ, Park Y, et al. Astaxanthin-Rich Extract from the Green Alga Haematococcus pluvialis Lowers Plasma Lipid Concentrations and Enhances Antioxidant Defense in Apolipoprotein E Knockout Mice. The Journal of Nutrition. 2011;141:1611-7.

[24] Kim D, Kim WR, Kim HJ, Therneau TM. Association between noninvasive fibrosis markers and mortality among adults with nonalcoholic fatty liver disease in the United States. Hepatology. 2013;57:1357-65.

[25] Kisseleva T, Brenner DA. Mechanisms of fibrogenesis. Experimental biology and medicine (Maywood, NJ). 2008;233:109-22. 
[26] Kisseleva T, Cong M, Paik Y, Scholten D, Jiang C, Benner C, et al. Myofibroblasts revert to an inactive phenotype during regression of liver fibrosis. Proc Natl Acad Sci U S A.

2012;109:9448-53.

[27] Friedman SL. Molecular regulation of hepatic fibrosis, an integrated cellular response to tissue injury. J Biol Chem. 2000;275:2247-50.

[28] Lee DH, Kim CS, Lee YJ. Astaxanthin protects against MPTP/MPP+-induced mitochondrial dysfunction and ROS production in vivo and in vitro. Food Chem Toxicol. 2011;49:271-80.

[29] Guerra BA, Otton R. Impact of the carotenoid astaxanthin on phagocytic capacity and ROS/RNS production of human neutrophils treated with free fatty acids and high glucose. Int Immunopharmacol. 2011;11:2220-6.

[30] Paik Y-H, Iwaisako K, Seki E, Inokuchi S, Schnabl B, Österreicher CH, et al. The nicotinamide adenine dinucleotide phosphate oxidase (NOX) homologues NOX1 and NOX2/gp91phox mediate hepatic fibrosis in mice. Hepatology. 2011;53:1730-41.

[31] Aoyama T, Paik YH, Watanabe S, Laleu B, Gaggini F, Fioraso-Cartier L, et al. Nicotinamide adenine dinucleotide phosphate oxidase in experimental liver fibrosis: GKT137831 as a novel potential therapeutic agent. Hepatology. 2012;56:2316-27.

[32] Katsuyama M. NOX/NADPH oxidase, the superoxide-generating enzyme: its transcriptional regulation and physiological roles. Journal of pharmacological sciences. 2010;114:134-46.

[33] Kaspar JW, Niture SK, Jaiswal AK. Nrf2:INrf2 (Keap1) signaling in oxidative stress. Free Radic Biol Med. 2009;47:1304-9.

[34] Ramani K, Tomasi ML, Yang H, Ko K, Lu SC. Mechanism and significance of changes in glutamate-cysteine ligase expression during hepatic fibrogenesis. J Bio Chem. 2012.

[35] Ryoo I-g, Ha H, Kwak M-K. Inhibitory Role of the KEAP1-NRF2 Pathway in TGF $\beta 1$ Stimulated Renal Epithelial Transition to Fibroblastic Cells: A Modulatory Effect on SMAD Signaling. PLoS ONE. 2014;9:e93265.

[36] Friedman SL. Mechanisms of hepatic fibrogenesis. Gastroenterology. 2008;134:1655-69.

[37] Iredale JP. Hepatic stellate cell behavior during resolution of liver injury. Semin Liver Dis. 2001;21:427-36.

[38] Blaner WS, O'Byrne SM, Wongsiriroj N, Kluwe J, D'Ambrosio DM, Jiang H, et al. Hepatic stellate cell lipid droplets: a specialized lipid droplet for retinoid storage. Biochim Biophys Acta. 2009;1791:467-73. 
[39] O'Byrne SM, Wongsiriroj N, Libien J, Vogel S, Goldberg IJ, Baehr W, et al. Retinoid absorption and storage is impaired in mice lacking lecithin:retinol acyltransferase (LRAT). J Biol Chem. 2005;280:35647-57.

[40] Yamaguchi K, Yang L, McCall S, Huang J, Yu XX, Pandey SK, et al. Diacylglycerol acyltranferase 1 anti-sense oligonucleotides reduce hepatic fibrosis in mice with nonalcoholic steatohepatitis. Hepatology. 2008;47:625-35.

[41] Cai K, Gudas LJ. Retinoic acid receptors and GATA transcription factors activate the transcription of the human lecithin:retinol acyltransferase gene. Int J Biochem Cell Biol. 2009;41:546-53. 


\section{Figure legends}

Figure 1. Experimental scheme for HSC activation and ASTX treatment. Mouse primary HSCs were activated on a plastic surface for 6 days and ASTX were added during the activation at D0, D2 and D4 until D6 (Design for ASTX effect on HSC activation). aHSCs (at D6 with no prior exposure to ASTX during activation) were also treated with ASTX for 2 or 4 additional days to address the effect of ASTX on inactivation of aHSCs (Design for ASTX effect on HSC inactivation).

Figure 2. Inhibition of HSC activation by ASTX. Mouse primary HSCs were activated for $6 \mathrm{~d}$ in the absence (control) or presence of $25 \mu \mathrm{M}$ ASTX from day 0, day 2 or day 4. (A) qRT-PCR analysis for $\alpha$-SMA mRNA abundance. Mean \pm SEM. $n=9$. Bars with a different letter within the same day are significantly different $(\mathrm{P}<0.05)$. $(\boldsymbol{B})$ A representative blot of $\alpha$-SMA protein of control and cells treated with ASTX from day 4 for $2 \mathrm{~d}$ during $6 \mathrm{~d}$ of activation.

Figure 3. Attenuated morphological changes of HSCs during activation by ASTX. After mouse primary HSCs were activated for $6 \mathrm{~d}$ in the absence (control) or presence of $25 \mu \mathrm{M}$ ASTX from day 0 , day 2 or day 4, cell morphology was determined at day 2, 4 and 6 (20x magnification). Black arrows indicate intracellular lipid droplets.

Figure 4. Reversal of aHSCs to an inactivated phenotype by ASTX. Mouse qHSCs were activated for $6 \mathrm{~d}$, and then incubated without (control) or with $25 \mu \mathrm{M}$ ASTX for 2 or $4 \mathrm{~d}$. (A) Cell morphology at day 8 or day 10 (20 x magnification). (B) qRT-PCR of controls or aHSCs treated with ASTX for 2 d. Mean \pm SEM. $n=6 .{ }^{*}, \mathrm{P}<0.05 .(\boldsymbol{C})$ Protein expression of $\alpha-S M A$ at 
day 8 in controls and cells treated with ASTX for 2 d. $(\boldsymbol{D})$ qRT-PCR of controls and cells treated with ASTX for $2 \mathrm{~d}$. Mean \pm SEM. $\mathrm{n}=6 . *, \mathrm{P}<0.05$.

Figure 5. The effect of ASTX on cellular ROS accumulation and NOX expression in mouse primary HSCs. (A) Cellular ROS levels were measured at day 6 in controls and ASTX-treated cells from day 0 for $6 \mathrm{~d}$. Data are expressed as arbitrary unit (fluorescent unit divided by total cell stain). (B) qRT-PCR analysis of controls or cells treated ASTX for $2 \mathrm{~d}$ from day 4. Mean \pm SEM. $n=6$.

Figure 6. Anti-fibrogenic activity of ASTX independent of NRF2. (A) Primary HSCs were isolated from wild-type and $\mathrm{Nrf}^{-/}$mice, and activated for $6 \mathrm{~d}$ from day 0 without (control) or with $25 \mu$ M ASTX for qRT-PCR. Mean \pm SEM. $n=9$. Bars with a different letter are significantly different $(\mathrm{P}<0.05)$. $(\boldsymbol{B})$ At day 6 of activation, HSCs were exposed to $3 \mu \mathrm{M}$ of tBHP with $25 \mu \mathrm{M}$ ASTX for $12 \mathrm{~h}$ for qRT-PCR analysis. Mean \pm SEM. $\mathrm{n}=6$. Bars with a different letter are significantly different $(\mathrm{P}<0.05)$. 
Figure 1
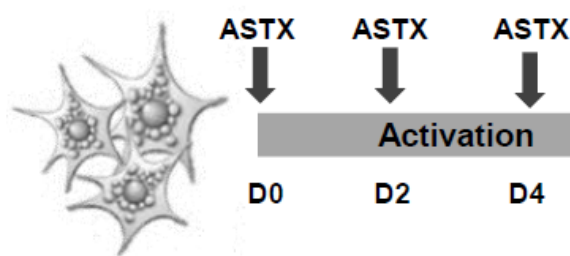

Quiescent HSC

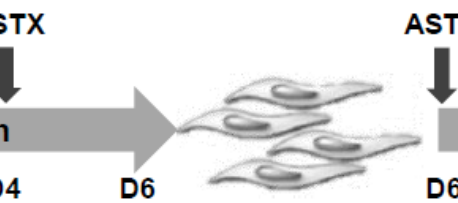

Activated HSC

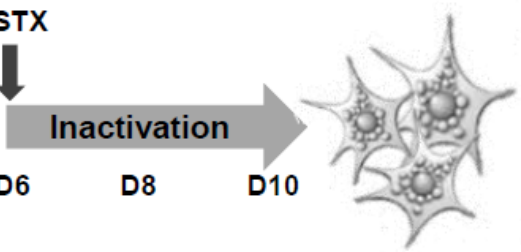

Inactivated HSC

Design for ASTX effect on HSC activation

Design for ASTX effect on HSC inactivation 
Figure 2

A

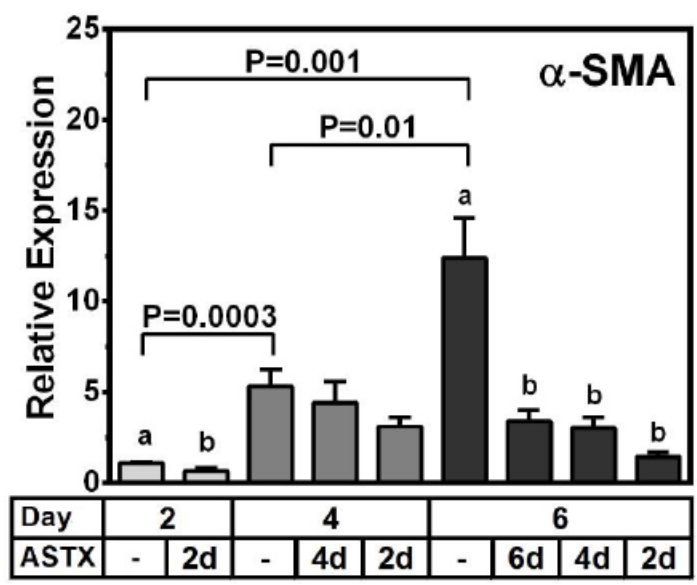

B

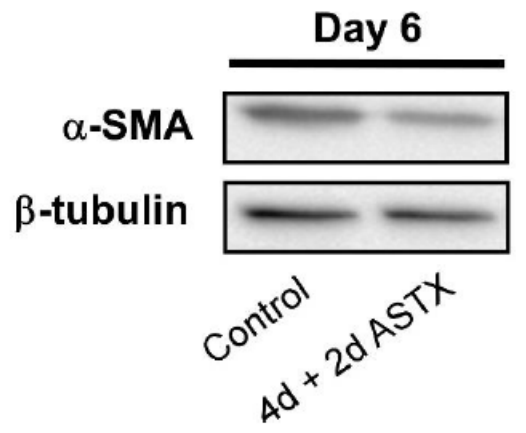


Figure 3

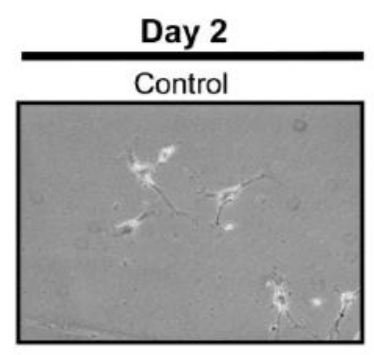

2d ASTX
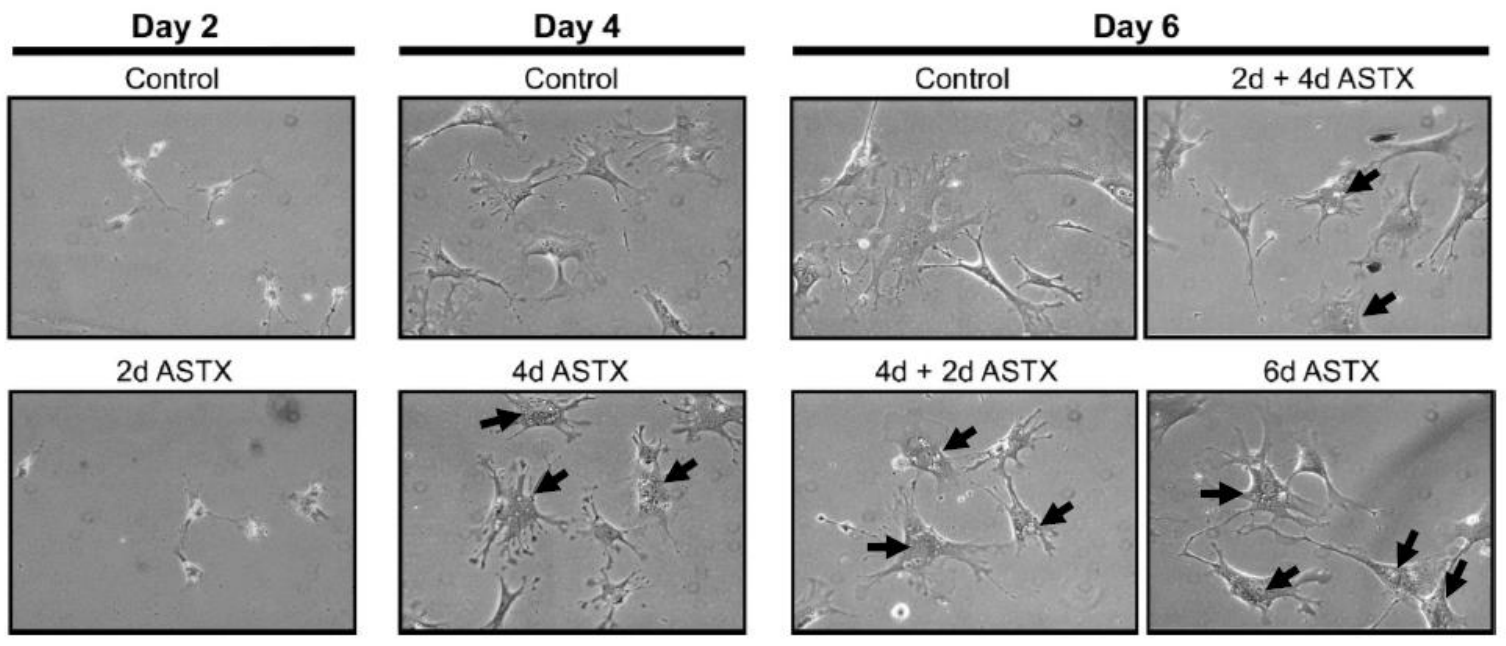
Figure 4

A
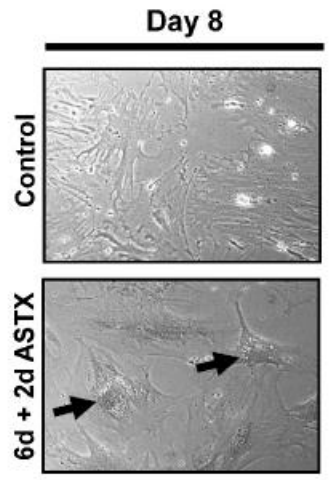

C

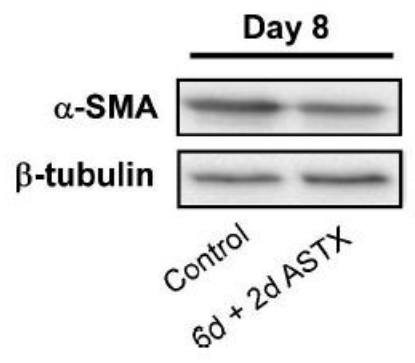

Day 10
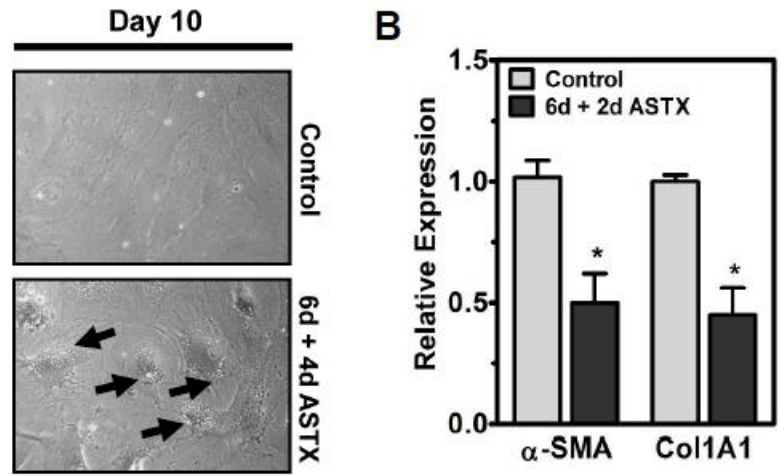

D

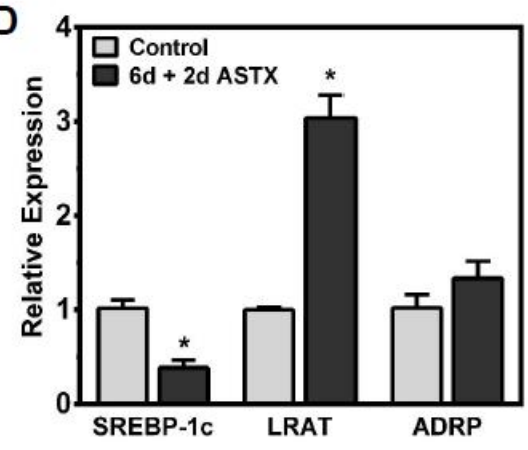


Figure 5
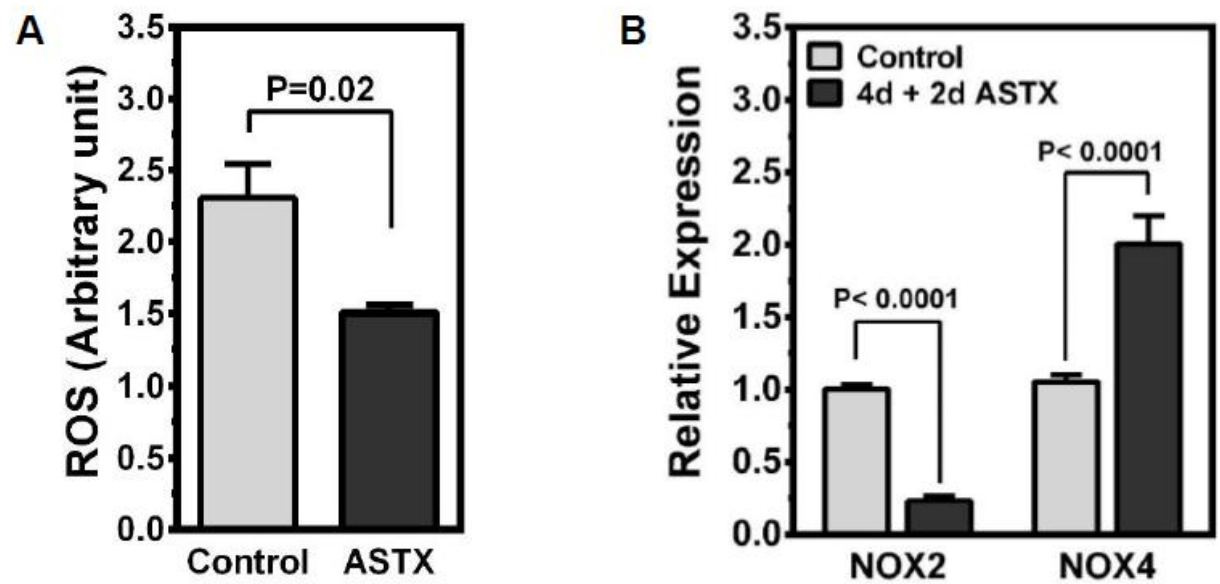
Figure 6
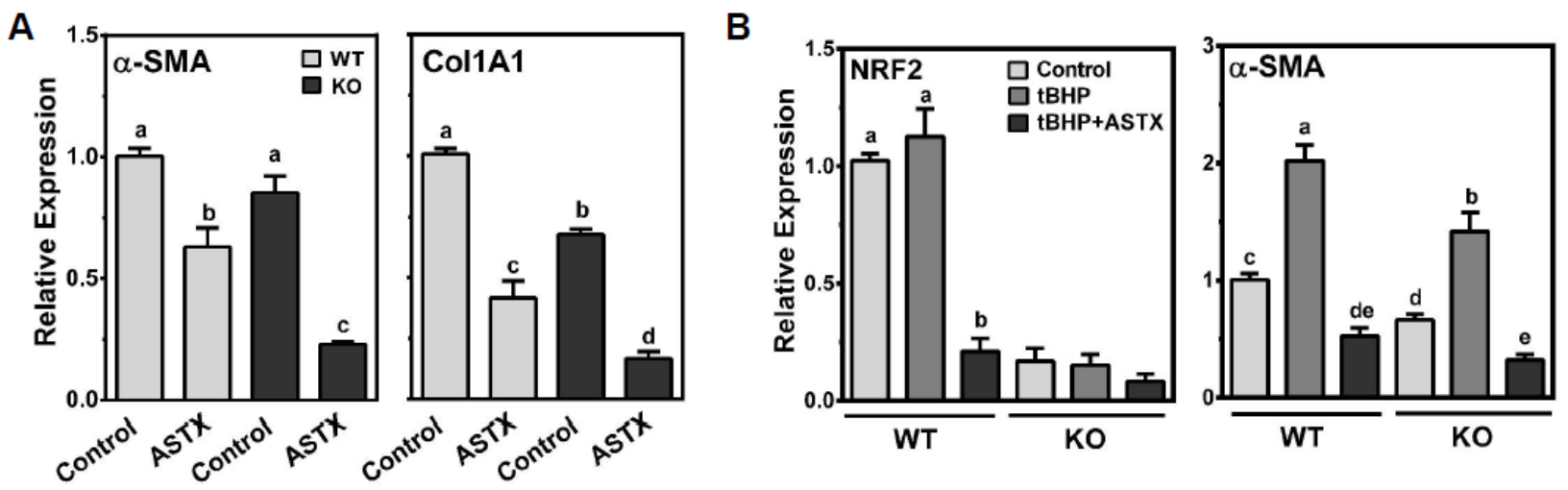